\title{
Ab Initio Study on the Electronic Band Structure, Density of States, Structural Phase Transition and Superconductivity of Zirconium
}

\author{
G.Jency ${ }^{*}$, S. Sutha Kumari \\ Research Centre \& Department of Chemistry, S.T.Hindu College, Nagercoil, TamilNadu, India \\ *Corresponding Author: jencygeorgejg@rediffmail.com
}

Copyright (C) 2013 Horizon Research Publishing All rights reserved.

\begin{abstract}
The electronic band structure, density of states, structural phase transition and super conducting transition temperature of group -IV B element Zirconium ( $\mathrm{Zr}$ ) under normal and high pressure are reported. The normal pressure band structure, density of states and superconducting transition temperature of hcp - $\mathrm{Zr}$ agree well with the previous calculations. The high pressure band structure exhibits significant deviations from the normal pressure band structure due to $s \rightarrow d$ transition. Zirconium undergoes structural phase transition from hexagonal closed packed (hcp) phase to body centered cubic (bcc) phase at 0.32 Mbar. For hcp $\rightarrow$ bcc transition, our calculated phase transition pressure is good agreement with the experimental result of $0.33 \mathrm{Mbar}$. According to the present calculation, at normal pressure, the superconducting transition of hep - Zr occurs at $0.53 \mathrm{~K}$ which is in agreement with the experimental observation of $0.55 \mathrm{~K}$. The density of states at the Fermi level $\left(N\left(E_{\mathrm{F}}\right)\right)$ is decreased as the pressure is further increased, which leads to the increase in the value of $T c(5.807 \mathrm{~K}$ at 0.32 Mbar).
\end{abstract}

Keywords Zirconium, Band Structure, Phase Transition

\section{Introduction}

Among the various transition metals and its alloys, group IV $\mathrm{B}$ transition metal Zirconium $(\mathrm{Zr})$ and its alloys are widely used as high temperature structural engineering materials in the aerospace industry. ${ }^{1}$ At ambient conditions Zirconium $(Z=40)$ crystallizes in a hep structure. ${ }^{2}$ When the temperature is raised above $1155 \mathrm{~K}$, its crystal structure changes to bcc. The band structure of $\mathrm{Zr}$ in hep structure was first obtained by Hugh et $a l^{3}$ and then by Jepsen ${ }^{4}$ using LMTO method. Later Bakonyi et al ${ }^{5}$ calculated the electronic structure and superconducting transition temperature $T_{c}$ for hcp-Zr and high temperature bcc-Zr using LMTO method.

There are many investigations both theoretical and experimental on phase transitions of $\mathrm{Zr}$ under pressure. ${ }^{6-15}$ Recently, the pressure induced structural transformation in $\mathrm{Zr}$ has received extensive experimental and theoretical attention. $^{2,16-20}$. However we are limiting our investigation only to the two phases (hcp and bcc ) of Zr. Here hcp is the normal pressure phase and bcc is the high temperature and high pressure phase. The present investigation is aimed at getting detailed information about the high pressure band structure (bcc - Zr), density of states and pressure variation of superconducting transition temperature. We have used the full potential linearized muffin tin orbital method (FP-LMTO)method ${ }^{21}$ and the details of the calculation are given in Ref.22 and will not be repeated here. The valence electronic configuration chosen in our calculations is $5 s^{2} 4 \mathrm{~d}^{2}$. The calculated total energies of hcp-Zr were fitted to Murnaghan's equation of $\operatorname{state}^{23}$ and the determined pressure values.

\section{Band Structure of Zirconium at Normal and High Pressure}

The band structures and density of states of $\mathrm{Zr}$ in the hep and bcc structures are obtained as a function of various reduced volumes (Figs.1 to 4). For hcp $-\mathrm{Zr}$ the normal pressure $\left(V / V_{o}=1\right)$ band structure is given in Fig.1. The overall topology of the band structure at $V / V_{o}=1$ is similar to previous calculations ${ }^{19}$. It is seen that the band structure exhibits characteristic features similar to other hep transition metals Ti and Hf. ${ }^{24,25}$ At normal pressure (Fig.1), there is a overlapping of valance band and conduction band confirming the metallic nature of $\mathrm{Zr}$. Similar to previous band structure calculations, ${ }^{19}$ in the present investigation also the conduction band minimum is located at the $\Gamma$ point. This feature is similar to $3 d$ transition metal titanium. The two bands lying well below the Fermi level are due to $5 s^{2}$ electrons and the bands near the Fermi energy are due to $4 d^{2}$ electrons.

As pressure increases, the entire band structure is slowly shifted up in energy and the conduction band width increases. 
The normal pressure conduction band width at $\Gamma$ point is equal to 0.3897 Ry (Fig.1) whereas it is 0.3469 Ry at 0.32 Mbar (Fig.3). The width of the conduction band decreases because of the enhanced overlap of the wave function with the neighboring atoms. The $s$ electron transfer to the $d$ shell under pressure is qualitatively a common feature of many band structure calculations. ${ }^{24,25}$ This increase in $d$ electron number leads to structural phase transition as well as superconducting transition under pressure. ${ }^{22}$

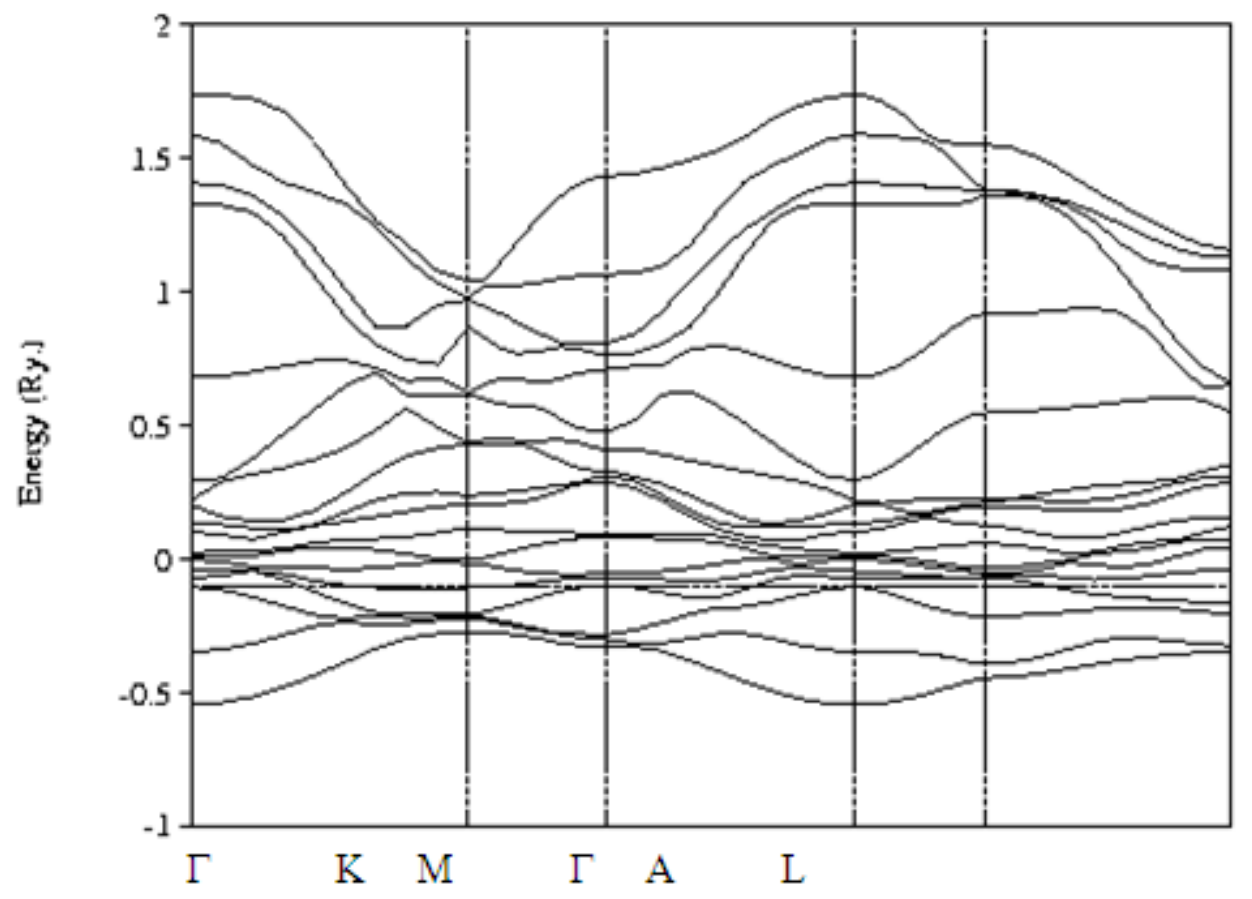

Figure 1. Band structure of hep- $\mathrm{Zr}$ at $\mathrm{V} / \mathrm{Vo}=1$ (normal pressure)

\section{Density of States of Zirconium at Normal and High Pressure}

The density of states (DOS) histogram constructed from the normal pressure band structure of hcp-Zr is given in Fig.2. Like in the previous calculations Fermi level $E_{F}$ lies in a minimum of the DOS curve (pseudo gap) (Fig.2). The levels arising from $4 d$ electrons give a high spike near $E_{F}$. The short and broad peak near -0.5 Ry is due to $5 s$ electrons. The highest peak with a broad width above the Fermi energy $E_{F}$ are due to empty $5 p$ and $6 s$ states. When pressure increases, the value of $E_{F}$ increases whereas density of states at $E_{F}$ decreases. ${ }^{24,25}$ It is due to the dispersion of the bands with pressure. The density of states (DOS) histogram constructed from the high pressure band structure of bcc-Zr is given in Fig.4.

These findings are in good agreement with previous TB-LMTO band structure calculations. The DOS values at $E_{F}$ are 32.29 states / Ry for bcc-Zr at 0.32 Mbar pressure (Fig.4) and 39.85 states / Ry for hcp-Zr at normal pressure (Fig.3). At high pressure, the $N\left(E_{F}\right)$ values of cubic bcc phase is smaller than hcp phase value. This is again in agreement with the results of previous calculations. The band structure results are used to calculate the superconducting transition temperature $T c$ in conjunction with McMillan's formula. The superconducting properties (to be discussed) are primarily determined by the $N\left(E_{F}\right)$ values around the Fermi level $E_{F}$ 

and Superconductivity of Zirconium

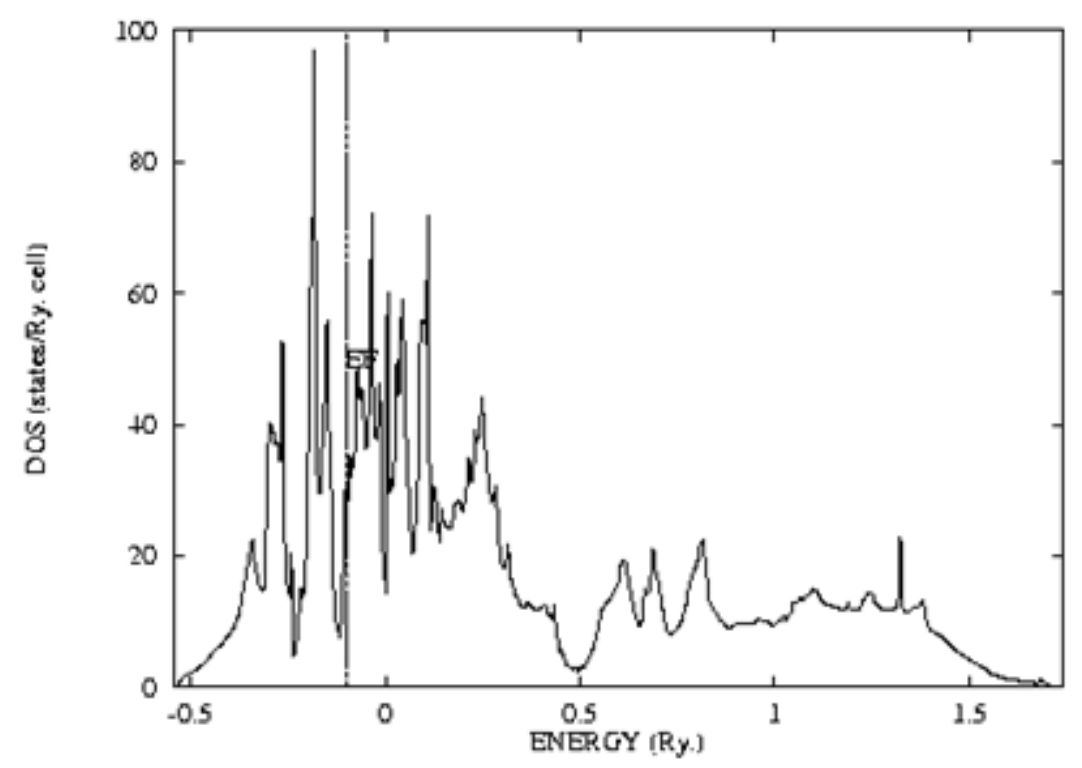

Figure 2. Density of states of hcp- $\mathrm{Zr}$ at $\mathrm{V} / \mathrm{Vo}=1$ (normal pressure)

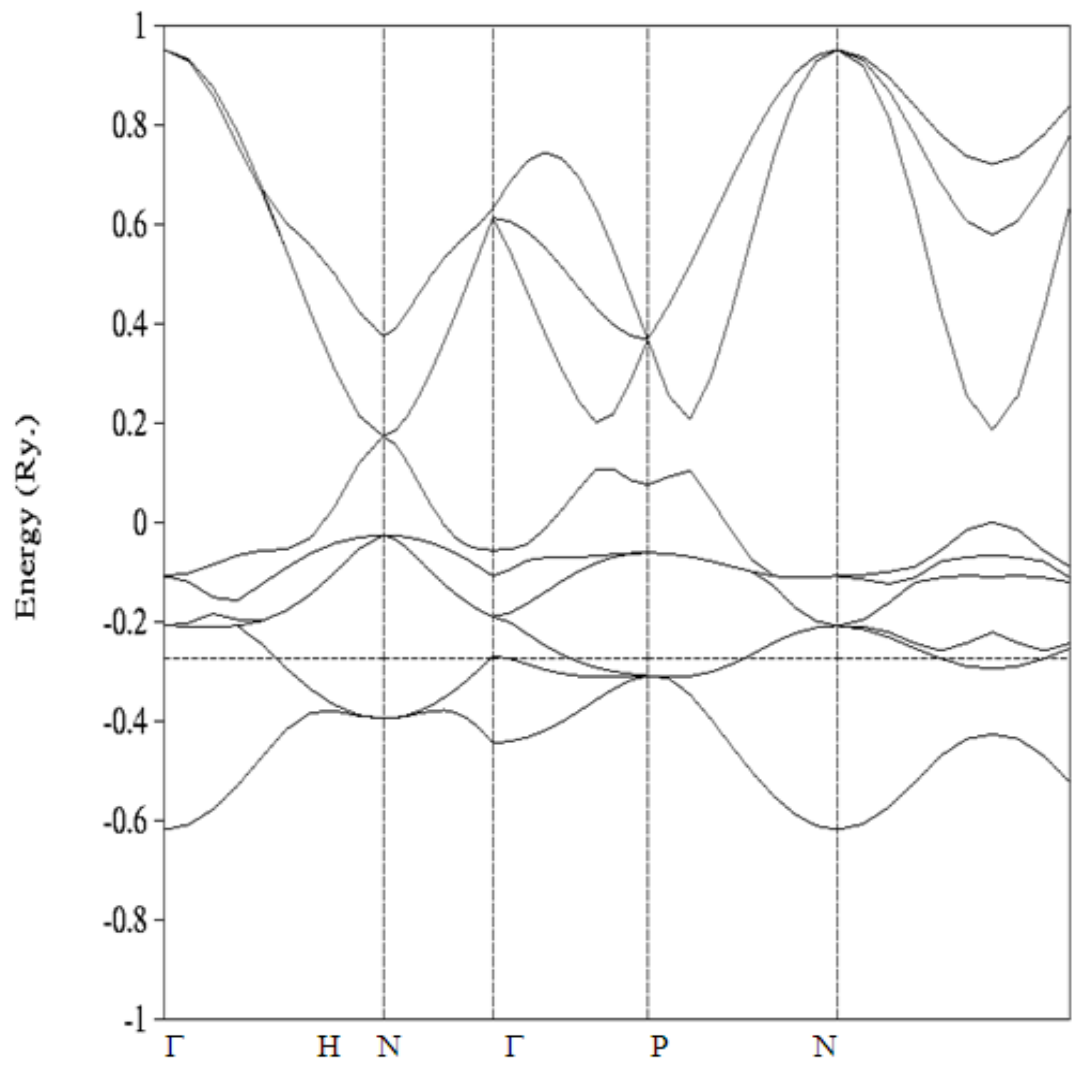

Figure 3. Band structure of bcc $-\mathrm{Zr}$ at $\mathrm{V} / \mathrm{Vo}=0.8(\mathrm{P}=0.32 \mathrm{Mbar})$ 


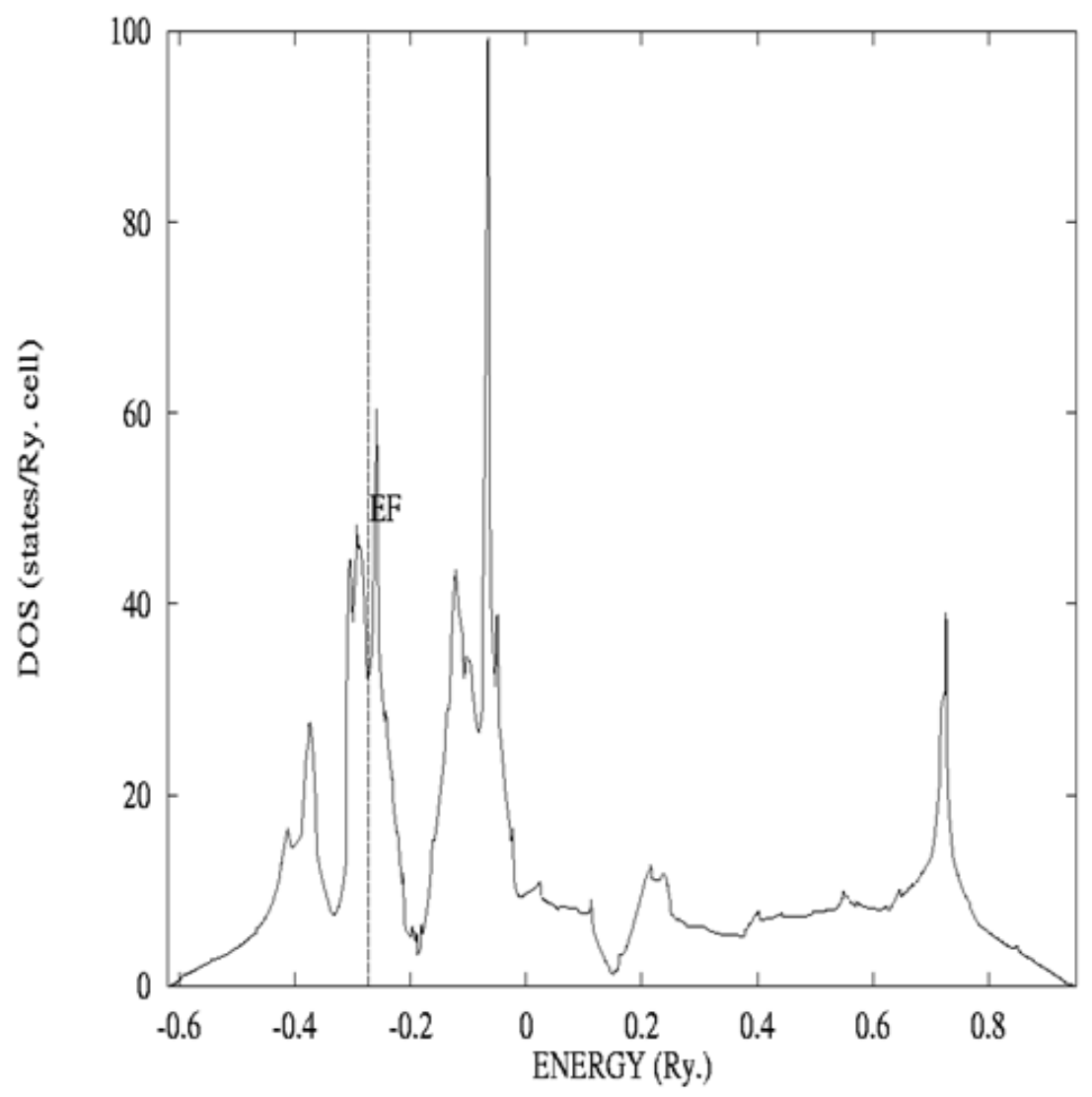

Figure 4. Density of states of bcc- $\mathrm{Zr}$ at $\mathrm{V} / \mathrm{Vo}=0.8(\mathrm{P}=0.32 \mathrm{Mbar})$

\section{Structural Phase Transition}

In our calculation the total energies as a function of reduced volume $\left(V / V_{\mathrm{o}}\right)$ are determined for hcp and bcc phases of $\mathrm{Zr}$ (Fig.5). Here $V / V_{\mathrm{o}}$ ranges from 1.2 to 0.4 insteps of 0.05 , where $V_{\mathrm{o}}$ is the experimental equilibrium volume and $V$ is any volume under pressure $P$. From the Fig.5, it is confirmed that the ground state structure of $\mathrm{Zr}$ is hcp as observed experimentally and reported from other theoretical calculations. The calculated total energies of hcp-Zr were fitted to Murnaghan's equation of state ${ }^{23}$ and the determined pressure values are given in Table 1. For the cubic structures (bcc) the total energies were calculated as a function of reduced volume $V / V_{o}$. For the hcp structures total energies were calculated as a function of $c / a$ and $V / V_{o}$. We performed calculations for different reduced volumes and different $c / a$ ratios in order to optimize both $V / V_{o}$ and $c / a$. In this calculation, the lattice constant and pressure of hcp $-\mathrm{Zr}$ at different reduced volume are calculated is shown in Table 1.

Table 1. Pressure And Lattice Constant of $\mathrm{Zr}$ at Different Reduced Volume

\begin{tabular}{|c|c|c|}
\hline V/Vo & $\begin{array}{c}\mathrm{a} \\
(\mathrm{au})\end{array}$ & $\begin{array}{c}\text { Pressure } \\
(\text { Mbar })\end{array}$ \\
\hline 1 & 6.6541 & 0 \\
0.9 & 6.4244 & 0.1178 \\
0.8 & 6.1771 & 0.3201 \\
0.7 & 5.9082 & 0.6506 \\
0.6 & 5.6122 & 1.2574 \\
0.5 & 5.2813 & 2.4304 \\
0.4 & 4.9027 & 4.9472 \\
0.3 & 4.4544 & 11.3027 \\
\hline
\end{tabular}




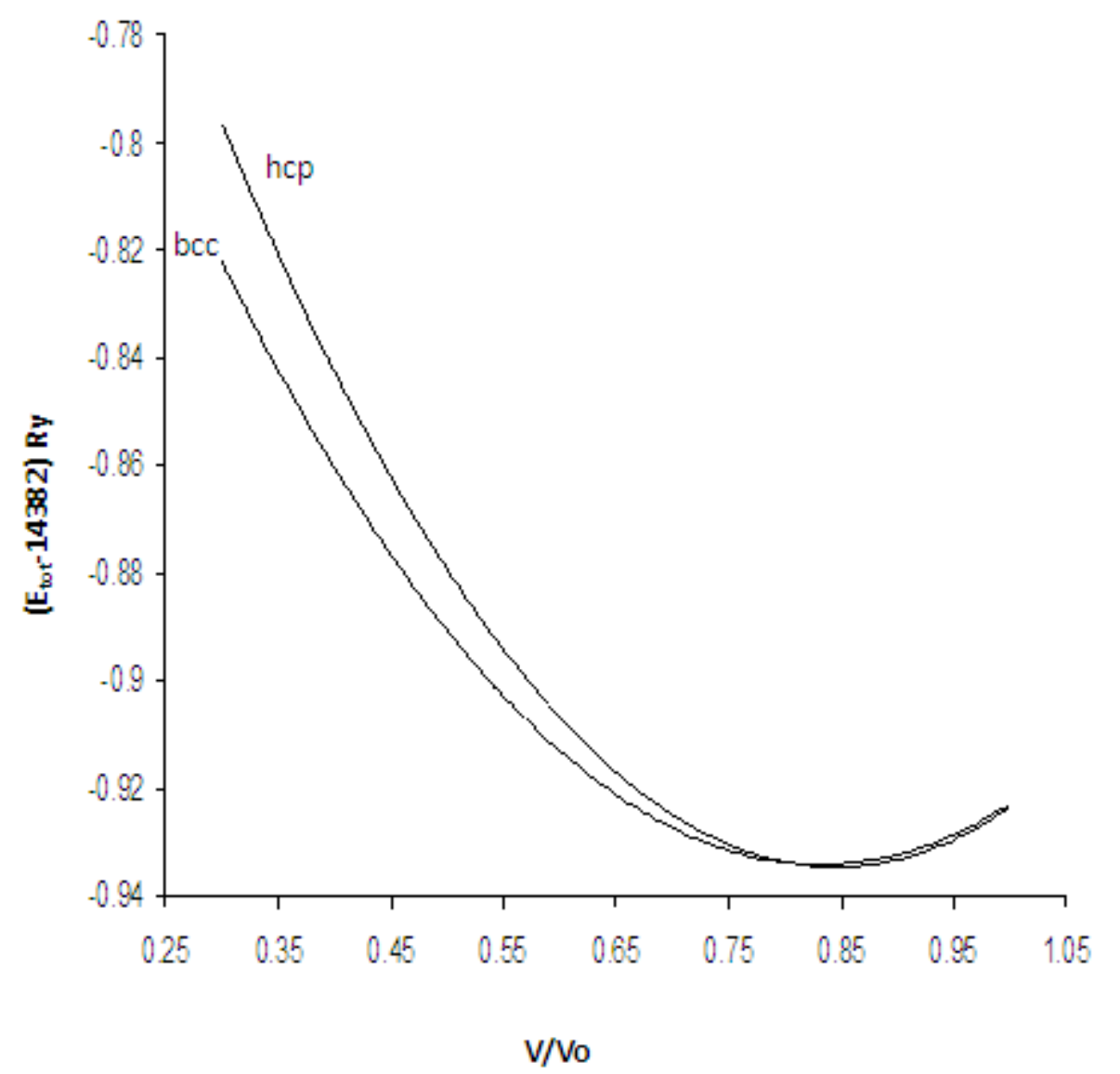

Figure 5. Total energy versus reduced volume curve of Zirconium

The calculated equilibrium lattice constants $\left(a_{o \&} c_{o}\right)$, bulk modulus $\left(B_{0}\right)$ and its pressure derivative $\left(B_{0}{ }^{1}\right)$ of $\mathrm{Zr}$ are given in Table.2. In this investigation, the total energies as a function of reduced volume (V/Vo) are determined for hcp, and the bcc phases of Zirconium are shown in Fig 5. The predicted ground state properties are in agreement with the previous TB model ${ }^{14}$ and experimental measurements. The pressure derivative of bulk modulus at normal pressure $B_{0}{ }^{1}$ is a parameter of great physical significance in high pressure physics and few other thermo-physical properties. ${ }^{22}$ The value of $B_{0}{ }^{1}$ is related to the electron density. The structure with highest $B_{0}{ }^{1}$ will have low electron density and vice versa. At ambient conditions, $\mathrm{Zr}$ has hep structure similar to other group IVA metals $\mathrm{Ti}$ and $\mathrm{Hf} .{ }^{24,25}$ Under pressure $\mathrm{Zr}$ undergoes hcp $\rightarrow$ bcc phase transitions. Based upon the recent experimental study, Akahama et $a l^{2}$ opinioned that there could be a possibility of bcc structure to exist above 0.33 Mbar and suggested that further experimental and theoretical investigations are needed in this direction. To ensure the high pressure structure of $\mathrm{Zr}$ above $0.33 \mathrm{Mbar}$, we have calculated the total energies corresponding to hep and bcc structures and the results are shown in Fig.5. Further from the enthalpy calculation, similar to titanium ${ }^{26,27}$ we have analyzed the structural phase transition from hcp $\rightarrow$ bcc structure. Further from the enthalpy calculation, we have analyzed the structural phase transition from hcp - bcc structure is shown in Table 3. The calculated values from this we get the phase transition pressure as 0.32 Mbar. Our calculated value for hcp $\rightarrow$ bcc transition is good agreement with the experimental value of Akahama et $\mathrm{al}^{2}$, the phase transition pressure for obtaining bcc structure is greater than 0.33 Mbar.

Table 2. Ground State Properties of Zirconium

\begin{tabular}{|c|c|c|c|c|}
\hline $\begin{array}{c}\text { Transition } \\
\text { Metal }\end{array}$ & $\begin{array}{c}\mathrm{a}_{\mathrm{o}} \\
(\mathrm{au})\end{array}$ & $\begin{array}{c}\mathrm{c}_{\mathrm{o}} \\
(\mathrm{au})\end{array}$ & $\begin{array}{c}\mathrm{B}_{\mathrm{o}} \\
(\mathrm{Mbar})\end{array}$ & $\mathrm{B}_{\mathrm{o}}{ }^{1}$ \\
\hline Zirconium & 6.6541 & 9.7354 & 0.911 & 3.895 \\
\hline
\end{tabular}

Table 3. Structural Phase Transition Pressure Of Zirconium

\begin{tabular}{|c|c|c|}
\hline \multirow{2}{*}{ Transition Metal } & \multicolumn{2}{|c|}{ Structural Phase Transition } \\
\cline { 2 - 3 } & $(\mathrm{V} / \mathrm{Vo})_{\mathrm{T}}$ & $\mathrm{P}_{\mathrm{T}}(\mathrm{Mbar})$ \\
\hline Zr (hcp- bcc) & 0.8 & 0.32 \\
\hline
\end{tabular}

\section{Superconducting Phase Transition}

The promotion of $s$ electron to $d$ shell in solids is one of the factors which will induce superconductivity. ${ }^{22}$ The manner in which the $d$ electron number is increasing as a function of pressure in hcp-Zr determines its electronic properties. At normal pressure itself the contribution of $4 d$ 
states is large. This clearly shows the possibility of a superconducting transition in hcp-Zr under normal pressure. A similar situation is observed in titanium.

The variation of $\theta_{D}$ with pressure can be estimated from a relation which is obtained assuming a free electron approximation. The rise in the Fermi energy with pressure in transition metals is in accordance with a free electron gas subjected to pressure. ${ }^{28}$ According to the free electron approximation, the Fermi energy is directly proportional to the electron concentration. This free electron concentration should increase with pressure which in turn increases the Fermi energy. The increasing electron concentration with pressure is directly proportional to the Fermi energy ${ }^{29}$ This Fermi energy fits well into the free electron based equation ${ }^{22}$

$$
\theta_{D}(P)=\frac{\sqrt{E_{F}}}{\sqrt{E_{F}^{o}}} \frac{a_{o}}{a} \theta_{D}^{o}
$$

where $\theta_{D}^{o}, a_{O}$ and $E_{F}^{o}$ are normal pressure quantities. For each pressure we get $\mathrm{E}_{\mathrm{F}}$ with reference to the constant potential outside which is reduced to zero to have a common reference. $\theta_{D}^{O}$ for hep $\mathrm{Zr}$ is $291 \mathrm{~K}^{29}$ The McMillan's formula, ${ }^{30}$ used for $T c$ calculation,

$$
T c=\frac{\theta_{D}}{1.45} \exp \left[\frac{-1.04(1+\lambda)}{\lambda-\mu^{*}(1+0.62 \lambda)}\right]
$$

gives the good estimate of the $T c$ value. Here $\mu^{*}$ is the electron-electron interaction parameter which is estimated using the relation, ${ }^{31}$

$$
\mu^{*}=\frac{0.26 N\left(E_{F}\right)}{1+N\left(E_{F}\right)}
$$

where $N\left(E_{F}\right)$ is the density of levels per atom per eV at $E_{F}$

With the results obtained from the self-consistent band structure calculation, we have computed $\theta_{D}, \lambda, \mu^{*}$ and $T c$ as a function of pressure. ${ }^{22}$ The calculated values at normal and various high pressures are given in Table.4 for hcp - Zr.

At normal pressure the $T c$ is $0.53 \mathrm{~K}$. This is in agreement with the experimental observation of $0.55 \mathrm{~K}^{32}$ The electron-phonon mass enhancement factor $\lambda$ and electron-electron interaction parameter $\mu^{*}$ at normal pressure are 0.41 and 0.13 respectively (Table.3), which are in agreement with the values of 0.40 and 0.13 respectively reported by McMillan. ${ }^{30}$ The increase of $T c$ in bcc-Zr at 0.32 Mbar $(5.807 \mathrm{~K})$ is due to the softening of phonon modes which arises because of lattice softening induced by $s \rightarrow d$ transition. ${ }^{33}$

In our calculation, hcp $\rightarrow$ bcc transition are obtained at $0.32 \mathrm{Mbar}$. The variation of $T c$ with pressure is computed for hcp and bcc structures and the results are given in Table.4. This increasing trend of $T c$ is similar to other group IVB transition metal $\mathrm{Ti}^{19}{ }^{19}$ At normal pressure the Debye temperature for the hcp phase $(291 \mathrm{~K})$ is higher than that of bcc phase $(200 \mathrm{~K})$ at $0.32 \mathrm{Mbar}$. Thus there is a softening of phonon in bcc phase. This phonon frequency softening (decrease of $\left\langle\omega^{2}\right\rangle$ ) contribute to a large $\lambda(0.88$ in Table 4$)$ and hence a high value of $T c$. The path to higher $T c$ lies in the direction of higher $\theta_{D}(P)$. But under very high pressure, a higher Debye temperature can also lower $T c$. That is because $\lambda$ can decrease if the phonon frequencies are large. The calculated $T c$ values depend more sensitively on changes in $\lambda$ and $\mu^{*}$ as these quantities appear with in the exponential term. ${ }^{33}$

Table 4. Superconducting Transition Temperature Of Hcp And Bcc Zirconium

\begin{tabular}{|c|c|c|c|c|}
\hline $\begin{array}{c}\text { Structure of } \\
\text { Zirconium }\end{array}$ & $\lambda$ & $\theta_{\mathrm{D}}(\mathrm{K})$ & $\mu$ & $\mathrm{Tc}(\mathrm{K})$ \\
\hline $\begin{array}{c}\text { hcp }-\mathrm{zr} \\
\text { (normal pressure) }\end{array}$ & 0.41 & 291 & 0.13 & 0.53 \\
\hline $\begin{array}{c}\mathrm{bcc}-\mathrm{zr} \\
(\mathrm{P}=0.32 \\
\text { Mbar })\end{array}$ & 0.88 & 200 & 0.17 & 5.807 \\
\hline
\end{tabular}

\section{Conclusion}

In summary, we have investigated the pressure dependent band structure and density of states of Zirconium using FP-LMTO method. The total energies of Zirconium are computed and the results are used to study the structural phase transition and superconductivity under pressure. The structural phase transition from hcp to bcc is found to occur at $0.32 \mathrm{Mbar}$ for Zirconium. Experimental phase transition pressure is 0.33 Mbar. Our calculated value is good agreement with the experimental value. At normal pressure the $T c$ is $0.53 \mathrm{~K}$. This is in agreement with the experimental observation of $0.55 \mathrm{~K}$. The increase of $T c$ in bcc-Zr at 0.32 Mbar $(5.807 \mathrm{~K})$ is due to the softening of phonon modes which arises because of lattice softening induced by $s \rightarrow d$ transition.

\section{REFERENCES}

[1] L.S.Dubrovinsky, N.A. Dubrovinskaia, V.Swamy, J. Muscat, N.M. Harrison, R.Ahuja, B.Holm and B. Johansson, Nature 410, 653 (2001)

[2] Y. Akahama, H. Kawamura and T.L. Bihan, J.Phys.: Condens. Matter 14, 10583 (2002).

[3] E.H. Hygh and R.M. Welch, Phys. Rev. B 1, 2424 (1970).

[4] O. Jepsen, Phys. Rev. B 12, 2988 (1975).

[5] I. Bakonyi, H. Ebert and A.I. Liechtenstein, Phys. Rev. B 48, 7841 (1993).

[6] J.C. Jamieson, Science 140, 72 (1963). 
[7] A. Jayaraman, W.Jr. Clement and G.C. Kennedy, Phys. Rev. 131, 644 (1963).

[8] R.G. McQueen, S.P. Marsh, J.W. Taylor, J.N. Fritz and W.J. Carter, High velocity Impact phenomena ed. R. Kinslow (New York: Academic, 1970) p.293.

[9] J.S. Gyanchandani, V. Vijayakumar. S.C. Gupta, S.K. Sikka, and R. Chidambaram, Proc.Solid state Phys. Symp. 31C, (Bombay: Dept. of Atomic Energy, 1988) p.279.

[10] H.Xia, G.Parthasarathy, H.Luo, Y.K. Vohra and A.L. Ruoff, Phys. Rev. B 42, 6736(1990).

[11] Y.K. Vohra, Acta Metall.27,1671 (1979).

[12] H.L. Skriver, Phys. Rev. B 31, 1909 (1985).

[13] J.S. Gyanchandani, S.C. Gupta, S.K. Sikka and R. Chidambaram, J.Phys.: Condens.Matter 2, 301 (1990).

[14] R.Ahuja and J.M. Wills, Phys. Rev. B 48, 16269 (1993).

[15] S.A. Ostanin and V.Yu Trubitsin, , J.Phys.: Condens. Matter 2, 301 (1990).

[16] Y.K.Vohra and P.T.Speneer, Phys. Rev. Lett. 86, 3068 (2001).

[17] K.D. Joshi, G. Jyoti, S.C. Gupta and S.K. Sikka, J.Phys.: Condens. Matter 14, 10921(2002).

[18] M.J. Mehl and D.A. Papaconstantopoules, Euro. Phys. Lett. 60,248 (2002)

[19] A. Aguago, G. Murrieta and R. De Coss, Phys. Rev. B 65, 092106 (2002).

[20] A.L. Kutepov and S.G. Kutepova, Phys. Rev. B 67, 132102 (2003).
[21] O. K. Andersen, O. Jepsen and M.Sob, in Elctronic band structure and its applications, edited by M. Yussouff (Springer, Heidelberg,1987) P.1 S.Limpijumnong and W.L.Lambrecht, Phys.Rev. B, 63, 104103 (2001).

[22] C.Nirmala Louis and K.Iyakutti, Phys. Rev. B 67, 094509 (2003); Phys. Rev.B.70, 132504 (2004).

[23] F.D. Murnaghan, Proc. Natl. Acad. Sci, U.S.A. 30, 244 (1944).

[24] M. Sanati, A. Saxena, T. Lookman and R.O. Albers, Phys. Rev. B 63, 224114 (2001).

[25] O.Le Bacq, F.Willaime and A. Pasturel, Phys. Rev. B 59, 8508 (1999).

[26] C.W. Greeff, D.R.Trinkle and R.C. Albers, J. Appl. Phys. 90, 2221 (2001).

[27] A.Heiming, W.Petry, J.Trampenau, M.Alba, C. Herzig, W. Mickeley and H.R.Vogl Phys. Rev. B 43, 10948 (1991).

[28] N. W. Ashcroft and N. D. Mermin, Solid State Physics (Holt Saunders, Tokyo, Japan,1981), p. 458.

[29] M.Dakshinamoorthy, K.Iyakutti, S.Sankar and R.Asokamani, Z.Phys.B: Condens.Matter 55, 299 (1984).

[30] W.L. McMillan, Phys. Rev. 167, 331 (1968).

[31] K. H. Bennemann and J. W. Garland, in Superconductivity in d- and f- Band Metals,edited by D.H. Douglas, AIP Conf. Proc. No. 4, (AIP, New York, 1972), p.103.

[32] B.W.Roberts, J.Phys.Chem. Ref. Data 5, 581 (1976).

[33] N.E. Christensen and D.L. Novikov, Phys.rev.B, 73, (2006) 224508. 